\title{
INTERACTION OF ATOMIC HYDROGEN WITH CARBON GRAINS
}

\author{
V. Mennella ${ }^{1}$
}

\begin{abstract}
Laboratory studies on the interaction of atomic hydrogen with aliphatic and aromatic hydrogenated carbon grains are discussed. When exposed to atomic hydrogen, both types of hydrogenated carbon grains act as catalysts for molecular hydrogen formation. In the first case, an exchange reaction with hydrogen chemisorbed in aliphatic carbon sites is the formation route to $\mathrm{H}_{2}$ formation. For aromatic carbon grains, the formation of molecular hydrogen takes place through a twostep reaction sequence: 1) super hydrogenation of the aromatic carbon islands of grains 2) exchange reactions on these islands. This mechanism represents a good approximation of molecular hydrogen formation on large neutral PAHs.
\end{abstract}

\section{Introduction}

The evolution of carbon dust in the Interstellar Medium (ISM) is driven by heat, irradiation by cosmic rays and UV photons and interaction with gas. Simulation of carbon dust processing under diffuse and dense medium conditions indicates that the key process for the evolution of the interstellar aliphatic carbon component is its interaction with $\mathrm{H}$ atoms. This interaction is able to counteract the destruction of $\mathrm{C}-\mathrm{H}$ bonds by UV photons and cosmic rays and activates the $3.4 \mu \mathrm{m}$ absorption band in diffuse interstellar regions and the $3.47 \mu \mathrm{m}$ feature in dense clouds (Mennella et al. 2001, 2002, 2003; Mennella 2008a). The interesting aspect is that the same carbon grain population can absorb at the two different wavelengths as a consequence of evolutionary transformations caused by processing. The transformations are compatible with the time-scale required by fast cycling of materials between dense and diffuse regions of the ISM. Moreover, this carbon component, after the inclusion in a comet during the formation process in the cold outer edge of the solar nebula, may evolve to develop the $\mathrm{CH}_{2}$ and $\mathrm{CH}_{3}$ groups (as suggested by laboratory simulations) and contribute to the aliphatic band at

${ }^{1}$ INAF-Osservatorio Astronomico di Capodimonte, Napoli, Italy 
$3.4 \mu \mathrm{m}$ of IDPs and in particles of comet Wild 2 collected by the Stardust mission (Mennella 2010).

The interaction of gas with dust grains also plays an important role for the chemical evolution of the ISM. Many molecules observed in the ISM can form as a consequence of gas-phase reactions (Herbst et al. 2005 and references therein). However, the formation of important species (e.g. $\mathrm{H}_{2}, \mathrm{H}_{2} \mathrm{O}$ and $\mathrm{CO}_{2}$ ) requires the presence of dust grain surfaces that act as catalysts. In the case of molecular hydrogen, gas-phase reactions for the conversion of $\mathrm{H}$ atoms to $\mathrm{H}_{2}$ are inefficient to account for the observed abundances of this molecule in the ISM (e.g. Williams 2005). The catalytic role of silicates, carbon grains and water ice has been demonstrated at low temperature through experiments performed by several groups (e.g. Pirronello et al. 1997, 1999; Manicò et al. 2001; Hornekaer et al. 2003; Perets et al. 2005). The recombination efficiency is high only at low temperatures. Laboratory results fail to explain $\mathrm{H}_{2}$ production at higher grain temperatures such those expected in photodissociation regions (e.g. Habart et al. 2004), since the residence time of $\mathrm{H}$ atoms in physisorption sites on the surface is too short for recombination to take place.

\section{$2 \quad \mathrm{H}_{2}$ formation on aliphatic hydrogenated carbon grains}

To explain $\mathrm{H}_{2}$ production at high grain temperatures, Cazaux \& Tielens (2004) proposed $\mathrm{H}$ atom recombination in chemisorption sites of carbon particles. Their model relies on the experimental and theoretical results obtained on aromatic structures such as graphite and coronene-like carbon clusters. $\mathrm{H}$ atoms chemisorbed in aliphatic carbon sites were neglected, since their binding energy was considered too high for those atoms to take part to molecular hydrogen formation. Recently, it has been shown experimentally that the formation of $\mathrm{H}_{2}$ in aliphatic sites of hydrogenated carbon grains occurs (Mennella 2008b). Exposure of fully hydrogenated carbon grains with $\mathrm{D}$ atoms and deuterated carbon grains with $\mathrm{H}$ atoms has been performed. Figure 1 shows the spectral effects induced by atoms at $300 \mathrm{~K}$ interacting with carbon grains at room temperature. The spectrum of hydrogenated carbon grains (Fig. 1A) is characterized by the $\mathrm{C}-\mathrm{H}$ stretching band at $3.4 \mu \mathrm{m}$. Increasing the D atoms fluence, the $\mathrm{C}-\mathrm{H}$ stretching feature decreases while the corresponding C-D feature increases. The analogous result is obtained when deuterated carbon grains are exposed to $\mathrm{H}$ atoms (Fig. 1B). In this case, the C-D feature decreases while the $\mathrm{C}-\mathrm{H}$ band increases when $\mathrm{H}$ atom fluence increases.

The reaction sequence governing the process is an exchange reaction: first, there is abstraction of hydrogen by a D atom with production of a HD molecule in gas phase; the second step is addition of D atom. HD molecules were detected with real-time mass spectroscopy. From the evolution with atom fluence of the stretching band intensity the abstraction and addition cross sections have been estimated. The small value of the abstraction cross section $\left(3 \times 10^{-18} \mathrm{~cm}^{2}\right)$ indicates that the formation of HD takes place through an Eley-Rideal process (see Mennella 2008b for more details). Moreover, abstraction is the rate limiting step of the reaction sequence, since the abstraction and addition cross sections 


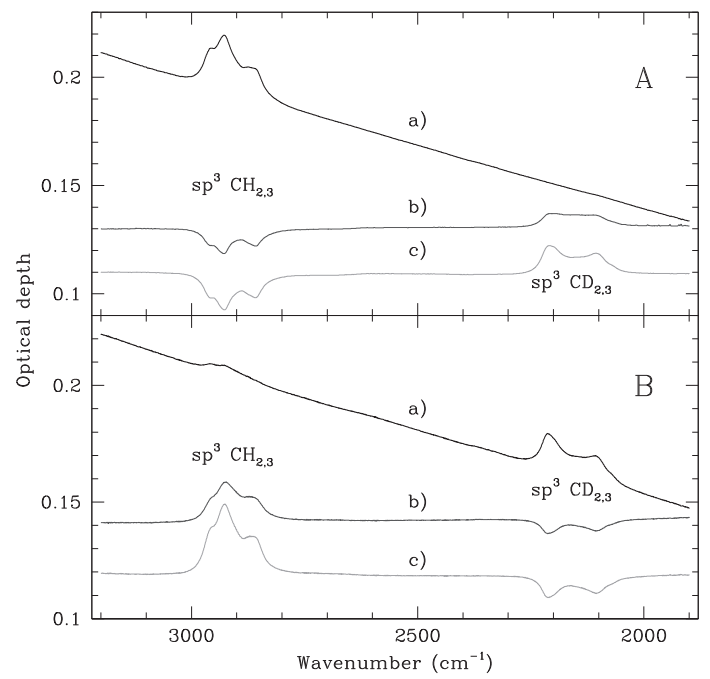

Fig. 1. Evolution of the C-H and C-D stretching bands during D irradiation of hydrogenated carbon grains (A) and $\mathrm{H}$ irradiation of deuterated carbon grains (B). In both panels the initial spectrum (a) and those after irradiation (shown after subtraction of the initial spectrum) of $6 \times 10^{17}$ (b) and $1 \times 10^{19}$ atoms $\mathrm{cm}^{-2}$ (c) are plotted. The difference spectra are offset for clarity. The weak sp ${ }^{3} \mathrm{CH}_{2,3}$ band present in the spectrum of deuterated carbon grains is due to the small fraction $(2 \%)$ of $\mathrm{H}_{2}$ present in the $\mathrm{D}_{2}$ gas used to produce the grains.

are equal, within the errors. These experimental results clearly suggest that $\mathrm{H}_{2}$ formation occurs on carbon grains even at high grain temperatures since $\mathrm{H}$ atoms are bonded to carbon with an energy of about $4 \mathrm{eV}$, and do not desorb even at high temperatures.

\section{$3 \quad \mathrm{H}_{2}$ formation on aromatic hydrogenated carbon grains}

Theoretical studies have suggested that $\mathrm{H}_{2}$ can form from abstraction of edge $\mathrm{H}$ atoms of PAH cations by incoming $\mathrm{H}$ atoms (Cassam-Chenai et al. 1994). Alternatively, it has been proposed that $\mathrm{H}_{2}$ can form on $\mathrm{PAH}$ cations in a twostep process: 1) an extra $\mathrm{H}$ atom is added to an edge site 2) abstraction of the excess $\mathrm{H}$ atom by a second incoming $\mathrm{H}$ atom (Bauschlicher 1998). In the case of cations, the formation does not have significant energy barriers. Theoretical studies have shown that low barrier routes to molecular hydrogen formation on neutral coronene exist: $\mathrm{H}_{2}$ should form by $\mathrm{H}$ abstraction on super hydrogenated coronene (Rauls \& Hornekaer 2008; Thrower et al., this volume). Laboratory experiments have shown that atomic and molecular hydrogen react with benzene and small PAHs (Petrie et al. 1992; Scott et al. 1997; Le Page et al. 1997; Snow et al. 1998). However, the formation of $\mathrm{H}_{2}$ has not been observed as yet. 


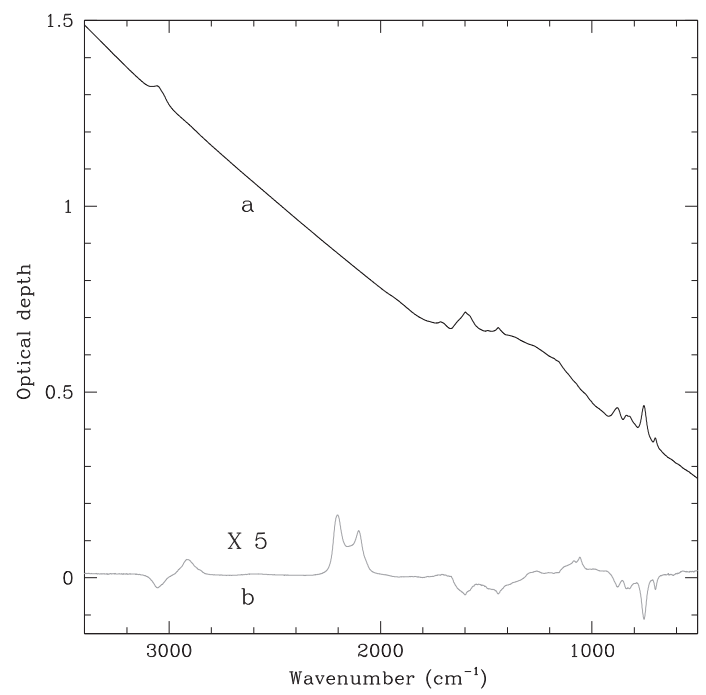

Fig. 2. IR spectrum of BE carbon grains as produced (a). The spectrum is characterized by the $\mathrm{C}-\mathrm{C}$ and $\mathrm{C}-\mathrm{O}$ bands at $1600 \mathrm{~cm}^{-1}(6.25 \mu \mathrm{m})$ and $1710 \mathrm{~cm}^{-1}(5.85 \mu \mathrm{m})$ respectively, the weak band at $1442 \mathrm{~cm}^{-1}(6.93 \mu \mathrm{m})$, and by the aromatic $\mathrm{C}-\mathrm{H}$ bands at $3050 \mathrm{~cm}^{-1}$ $(3.28 \mu \mathrm{m})$ (stretch), the weak mode at $1157 \mathrm{~cm}^{-1}(8.64 \mu \mathrm{m})$ (in plane bend) and the out of plane bend modes between 930 and $670 \mathrm{~cm}^{-1}$. The spectrum after irradiation (shown after subtraction of the initial spectrum) of $1.65 \times 10^{19} \mathrm{D}$ atoms $\mathrm{cm}^{-2}$ (b) is also plotted. The intensity of the C-C and C-H bands decreases while the aliphatic C-D stretch bands, falling between 2280 and $2000 \mathrm{~cm}^{-1}$, and the corresponding bending modes, between 1120 and $1020 \mathrm{~cm}^{-1}$, develop with D exposure.

Recently, the interaction of $\mathrm{H}$ atoms with a carbon soot (BE) with a marked aromatic character has been studied experimentally (Mennella, in preparation). $\mathrm{BE}$ grains have been produced by burning benzene $\left(\mathrm{C}_{6} \mathrm{H}_{6}\right)$ in air; they are characterized by a size distribution of ringed $\mathrm{sp}^{2}$ islands (e.g. Colangeli et al. 1995). The infrared spectrum of BE (Fig. 2) indicates that, with the exception of the features at $6.25 \mu \mathrm{m}$ and $5.85 \mu \mathrm{m}$, all the bands are due to $\mathrm{C}-\mathrm{H}$ vibrations of hydrogen bonded to aromatic carbon sites. There is no evidence for aliphatic C-H vibrational modes.

Exposure of $\mathrm{BE}$ at room temperature to $\mathrm{D}$ atoms at $300 \mathrm{~K}$ induces significant changes in the spectrum (Fig. 2). The C-C feature at $6.25 \mu \mathrm{m}$ and all the bands due to the aromatic $\mathrm{C}-\mathrm{H}$ modes decrease in intensity with $\mathrm{D}$ fluence. The formation of the C-D aromatic bands is not observed, while the aliphatic C-D bands develop with D irradiation. The feature at $2912 \mathrm{~cm}^{-1}$ is attributed to the $\mathrm{C}-\mathrm{H}$ stretching mode of CHD. The detailed evolution with $\mathrm{D}$ atom fluence of the aromatic $\mathrm{C}-\mathrm{H}$ and aliphatic C-D stretching band intensity is shown in Figure 3. From the observed trends, it is possible to derive the destruction cross section of aromatic $\mathrm{C}-\mathrm{H}$ bonds and the formation of the aliphatic $\mathrm{C}-\mathrm{D}$ bonds. The small 


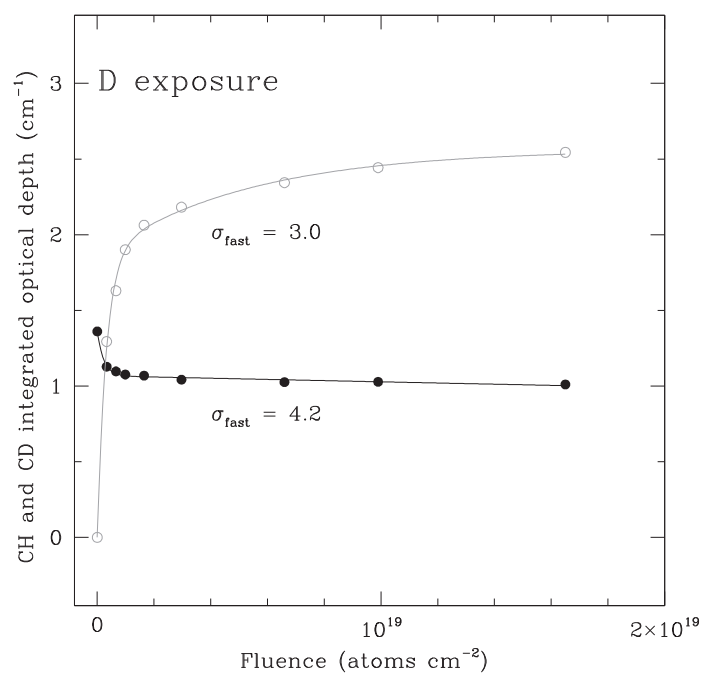

Fig. 3. Evolution with D atom fluence of the aromatic C-H (filled circles) and aliphatic C-D (empty cicles) stretch band integrated optical depth. The best fit to the data of the relations $a_{1}\left[1-\exp \left(-\sigma_{\text {fast }} F\right)\right]+a_{2}\left[1-\exp \left(-\sigma_{\text {slow }} F\right)\right]$ and $b_{1} \exp \left(-\sigma_{\text {fast }} F\right)+$ $b_{2} \exp \left(-\sigma_{\text {slow }} F\right)$, respectively for the $\mathrm{C}-\mathrm{D}$ and $\mathrm{C}-\mathrm{H}$ modes, are also shown. The estimated cross sections are expressed in units of $10^{-2} \AA^{2}$.

values of the cross sections suggest that an Eley-Rideal type process drives the spectral changes produced by D atom irradiation. Unlike the case of D irradiation of aliphatic hydrogenated carbon grains, the spectral changes do not suggest an exchange reaction in the aromatic sites, since the formation of aromatic C-D bonds is not observed. Rather, they indicate super hydrogenation of the aromatic structures of grains as new aliphatic C-D bonds form. In fact, D atoms break a $\mathrm{sp}^{2}$ double bonds inducing the formation $\mathrm{sp}^{3} \mathrm{C}-\mathrm{D}$ bonds.

As one can see in Figure 3, at high fluences the spectral variations are smaller and smaller. To understand which reactions are active in those conditions, at the end of $\mathrm{D}$ atom exposure of $\mathrm{BE}$, irradiation has been continued using $\mathrm{H}$ atoms at $300 \mathrm{~K}$. H atom exposure determines an increase of the aliphatic $\mathrm{C}-\mathrm{H}$ stretching and bending modes and a decrease of the corresponding aliphatic C-D bands. On the contrary, there is no variation of the aromatic features. These spectral changes are similar to those observed during $\mathrm{H}$ atom irradiation of deuterated aliphatic carbon grains and are indicative of exchange reactions in the aliphatic sites. The exchange reactions form HD molecules. These results clearly show that superhydrogenated aromatic carbon islands act as catalysts for molecular hydrogen formation. Moreover, they represent a good approximation of $\mathrm{H}_{2}$ formation on neutral PAHs, as the reactions induced by $\mathrm{H}$ atoms in aromatic carbon grains are Eley-Reideal type processes. 


\section{References}

Bauschlicher, C.W., Jr., 1998, ApJ, 509, L125

Cassam-Chenaï, P., Pauzat, F., \& Ellinger, Y., 1994, Molec. Grains Space, 312, 543

Cazaux, S., \& Tielens, A.G.G.M., 2004, ApJ, 604, 222

Colangeli, L., Mennella, V., Palumbo, P., Rotundi, A., \& Bussoletti, E., 1995, A\&AS, 113,561

Habart, E., Boulanger, F., Verstraete, L., Walmsley, C.M., \& Pineau des Forêts, G., 2004, A\&A, 414, 531

Herbst, E., Chang, Q., \& Cuppen, H.M., 2005, J. Phys. Conf. Ser., 6, 18

Hornekær, L., Baurichter, A., Petrunin, V.V., Field, D., \& Luntz, A.C., 2003, Science, 302, 1943

Le Page, V., Keheyan, Y., Bierbaum, V.M., \& Snow, T.P., 1997, J. Am. Chem. Soc., 119,8373

Manicò, G., Ragunì, G., Pirronello, V., Roser, J.E., \& Vidali, G., 2001, ApJ, 548, L253

Mennella, V., 2010, ApJ, 718, 867

Mennella, V., 2008a, ApJ, 682, L101

Mennella, V., 2008b, ApJ, 684, L25

Mennella, V., Baratta, G.A., Esposito, A., Ferini, G., \& Pendleton, Y.J., 2003, ApJ, 587, 727

Mennella, V., Brucato, J.R., Colangeli, L., \& Palumbo, P., 2002, ApJ, 569, 531

Mennella, V., Muñoz Caro, G., Ruiterkam, R., et al., 2001, A\&A, 367, 355

Perets, H.B., Biham, O., Manicó, G., et al., 2005, ApJ, 627, 850

Petrie, S., Javahery, G., \& Bohme, D.K., 1992, J. Am. Chem. Soc., 114, 9205

Pirronello, V., Biham, O., Liu, C., Shen, L., \& Vidali, G., 1997, ApJ, 483, L131

Pirronello, V., Liu, C., Roser, J.E., \& Vidali G., 1999, A\&A, 344, 681

Rauls, E., \& Hornekær, L., 2008, ApJ, 679, 531

Scott, G.B., Fairley, D.A., Freeman, C.G., et al., 1997, J. Phys. Chem. A, 101, 4973

Snow, T.P., Le Page, V., Keheyan, Y., \& Bierbaum, V.M., 1998, Nature, 391, 259

Williams, D.A., 2005, J. Phys. Conf. Ser., 6, 1 